\title{
Comparison of the biocompatibility of calcium silicate-based materials to mineral trioxide aggregate: Systematic review
}

\author{
Natália Gomes de Oliveira ${ }^{1}$, Pollyana Rodrigues de Souza Araújo ${ }^{1}$, \\ Marina Torreão da Silveira ${ }^{1}$, Ana Paula Veras Sobral ${ }^{2}$, \\ Marianne de Vasconcelos Carvalho ${ }^{1}$
}

Correspondence: Dr. Natália Gomes De Oliveira Email: nataliagomes04@hotmail.com
'Department of Dentistry and Endodontics, Faculty of Dentistry, University of Pernambuco, Camaragibe, PE, Brazil,

2Department of Patology, Faculty of Dentistry, University of Pernambuco, Camaragibe, PE, Brazil

\section{ABSTRACT}

The objective of this systematic review was to evaluate the biocompatibility and interaction of bioceramic materials with animal and human mesenchymal cells in vitro and in vivo and to compare them with mineral trioxide aggregate (MTA). Two independent researchers conducted PubMed/Medline, Web of Science, and Scopus searches to identify studies published in English, without restrictions on year of publication using the following keywords: "root canal sealer," "root repair material," "cytotoxicity," and "bioceramics." The articles were selected following the PRISMA statement. A total of 1486 titles were identified in the initial search. However, only 18 studies met the inclusion and exclusion criteria. The results showed that bioceramic materials have biological properties similar to those of MTA, including low cytotoxicity as well as promoting cell proliferation and adhesion, low expression of inflammatory cytokines, and reduced pulp inflammation. This systematic review therefore suggests that the choice of repair bioceramic materials or MTA based on biocompatibility should be the professional's decision.

Key words: Bioceramics, cytotoxicity, root canal sealer, root repair material

\section{INTRODUCTION}

Complete filling of root canal systems after chemo-mechanical preparation is critical to the success of endodontic treatment, as well as to sealing of the root ape ${ }^{[1]}$ since many materials exhibit limited contact with vital tissues in the apical region. However, in some procedures such as pulp capping/pulpotomy, perforation repair, apexification, and obturation itself, the materials are placed in proximity to pulp and apical periodontal tissues. ${ }^{[2]}$ Root repair materials

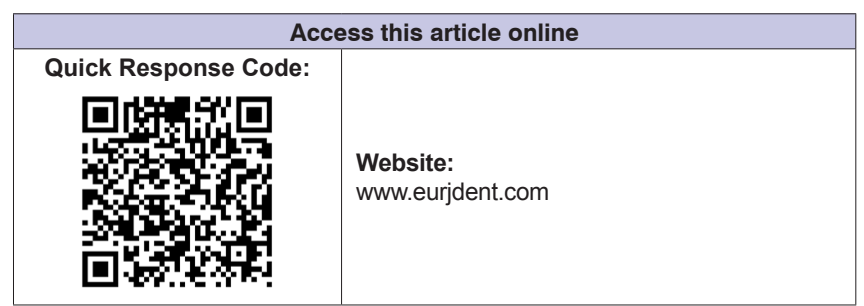

must therefore have excellent characteristics such as biocompatibility and stability of physical and chemical properties. In addition to these characteristics, the repair material should stimulate tissue regeneration, especially after endodontic treatment or apical pathology. ${ }^{[3,4]}$

Mineral trioxide aggregate's (MTA's) success as a repairing material is undeniable, although there are

This is an open access journal, and articles are distributed under the terms of the Creative Commons Attribution-NonCommercial-ShareAlike 4.0 License, which allows others to remix, tweak, and build upon the work non-commercially, as long as appropriate credit is given and the new creations are licensed under the identical terms.

For reprints contact: reprints@medknow.com

How to cite this article: de Oliveira NG, de Souza Araújo PR, da Silveira MT, Veras Sobral AP, Carvalho Md. Comparison of the biocompatibility of calcium silicate-based materials to mineral trioxide aggregate: Systematic review. Eur J Dent 2018;12:317-26.

DOI: 10.4103/ejd.ejd_347_17 
some limitations regarding its use. Limitations of MTA include color alteration, manipulation difficulties, the need of specific instruments, and delayed setting time. A reduction of the setting period has a beneficial effect on patient's relief and on bacterial infection. ${ }^{[5]}$ Based on these limitations, it is necessary to search for new materials that have better properties.

Bioceramic root canal sealers have recently been introduced into endodontics, with the same indications of the MTA, that is, or use in obturation and repair procedures. These cement contain tri- and di-calcium silicate, calcium phosphate, calcium hydroxide, as well as zirconium oxide as radiopacifier. ${ }^{[6]}$ Bioceramic materials are indicated as an alternative to MTA, due to their excellent physical, chemical, and biological properties, for example, they have been shown to induce cell differentiation, to have osteoconductive effects, and to reduce inflammation. ${ }^{[7,8]}$

The objective of this systematic review was to evaluate the biocompatibility and interaction of bioceramic materials with animal and human mesenchymal cells in vitro and in vivo and to compare them with MTA, since there are no randomized clinical trials that perform this type of comparison. Since it is a relatively new material in the endodontic market, it is necessary to compare its biocompatibility with materials such as MTA, which are considered the gold standard of endodontics. The hypothesis tested is that bioceramic materials are more biocompatible than MTA.

\section{METHODOLOGY}

\section{Procedure}

This systematic review was conducted according to the guidelines of thePRISMA statement. Thereview protocol was registered at PROSPERO (CRD42017056232). The studies were selected according to the inclusion and exclusion criteria reported below. All abstracts and full texts were reviewed. None of the manuscript author was contacted during this process. Disagreements between authors were evaluated and the studies were eliminated through discussion among researchers until a consensus was reached.

\section{Inclusion and exclusion criteria}

The inclusion criteria for this review included studies published in English, without restrictions on year of publication, and studies which evaluated biocompatibility, comparing the cytotoxicity of bioceramic materials to MTA. The types of studies were in vitro and in vivo laboratory studies using animal (no species restriction, either small or large) and human cells, prospective studies, retrospective studies, and randomized clinical trials. Excluded were studies that compared the cytotoxicity of bioceramic materials only, studies that compared bioceramic materials with cement other than MTA, or studies comparing bioceramic endodontic sealers, since the biocompatibility requirements of a repairing material are much greater than that of a sealer.

\section{Criteria for selection of the studies}

First, studies were selected by analysis of the titles. If the title indicated inclusion, the abstract was evaluated carefully and articles considered eligible for review (or in case of doubt) were selected for reading. Disagreements among researchers were resolved by discussion with a third researcher (MVC). The kappa level of agreement between authors was 0.81 . Due to thelack of randomized clinical trials and prospective and retrospective studies, this review included in vitro studies using animal and human cells and in vivo animal studies. For this reason, the patient-intervention-comparison-outcome (PICO) system was adapted: population (studies that evaluated animal and human mesenchymal cells), intervention (evaluation of the biocompatibility of bioceramic materials), comparison (MTA), and outcomes (cell viability, changes in cell morphology, inflammatory responses, cytokine production, and cell adhesion).

\section{Search strategy}

Two independent researchers (NGO and PRSA) conducted searches in PubMed/Medline, Web of Science, and Scopus to identify studies published in English without restriction on year of publication. The keywords used were "root repair material," "root canal sealer," "cytotoxicity," and "bioceramics." The search details were Root repair material (all fields) AND root canal sealer (all fields) OR (root repair material [all fields] AND cytotoxicity [All Fields]) OR root repair material (all fields) AND bioceramcis (all fields) OR root canal sealer (all fields) AND cytotoxicity (all fields) OR root canal sealer (all fields) AND bioceramics (all fields) OR cytotoxicity (all fields) AND bioceramics (all fields). This electronic search was complemented by a manual search conducted from March 1, 2016, to January 8, 2017, in high-impact journals in endodontics, such as the Journal of Endodontics and International Endodontics Journal.

\section{Assessment of risk of bias}

Since no specific evaluation exists for in vitro studies, this review critically evaluated the selected studies 
using an adapted version of the CONSORT checklist, which consists of 25 items; however, only 15 items were used in this study (presence of the first author's name, type of study being identified in the title, presentation of a structured abstract, introduction containing a scientific context, clearly describing the rationale, objectives, and hypothesis tested, methodology showing study type, cell type, intervention, statistical analysis, evaluation time between groups studied, if materials were used according to the manufacturers, main results from each experiment being described, if in the discussion section the results and their clinical implications were interpreted, whether these results could be translated into other species or systems, relevance to human biology, and the existence of funding. If the authors reported the information analyzed, a yes $(Y)$ answer was assigned to the specific parameter. If the information could not be found, a no (N) answer was given. Articles reporting 1-5 items were classified as high risk, 6-10 as medium risk, and 11-15 as low risk of bias. All the 18 presented low risk of bias.

\section{RESULTS}

The flowchart of the systematic review is depicted in Figure 1. A total of 1486 titles were identified in the initial search. Eighteen studies were included in this review and processed for data extraction in the following order: first author, year of publication, type of study (in vitro or in vivo), type of sealer, type of laboratory analysis, type of biocompatibility test, type of cell used (animal or human), incubation time or experimental period, and the main results found in each study according to the methodology applied [Table 1].

As a response to the possible results established in PICO, in general, the bioceramic materials exhibited similar biological properties when compared to MTA, including good biocompatibility, cell proliferation and adhesion, low cytotoxicity, low expression of inflammatory cytokines, and reduced inflammation of human pulp cells.

Eight (44\%) of the eighteen studies analyzed used the MTT or MTS assay for laboratory analysis [Table 1]. Only one study ${ }^{[18]}$ evaluated tissue reactions through histological analysis in subcutaneous tissues of rats (Wistar rats).

Eleven studies evaluated cytotoxicity, in which, in eight studies, the cytotoxicity of bioceramic materials

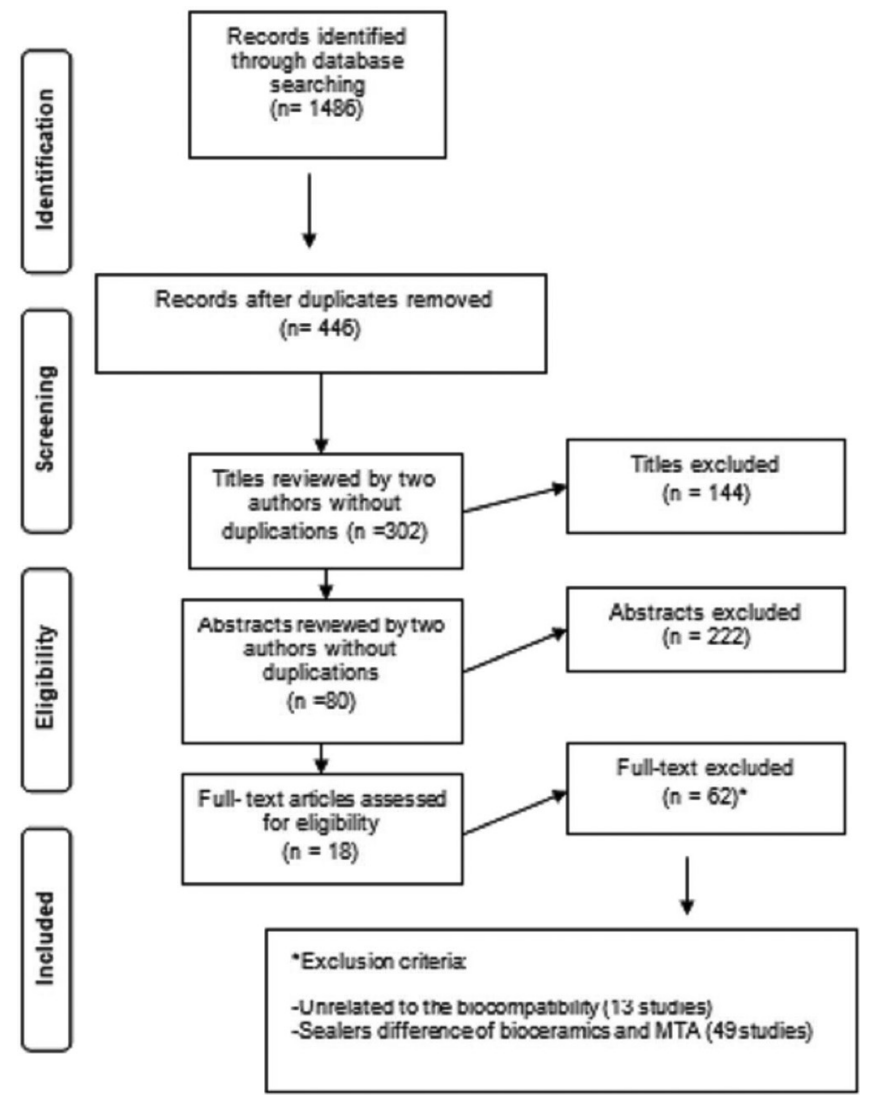

Figure 1: The flow chart

was similar to that of MTA. ${ }^{[9,10,13,15,16,20,22,24]}$ In one study, the results obtained with the bioceramic materials were superior. ${ }^{[12]}$ In contrast, two studies demonstrated greater biocompatibility of the MTA ${ }^{[21,23]}$ [Table 1].

Two of the studies included in this review evaluated the inflammatory responses and production and expression of cytokines (interleukin [IL]-1b, tumor necrosis factor-alpha [TNF- $\alpha$ ], IL-6, and IL-8) induced by these materials when in contact with mesenchymal cells. ${ }^{[13,17]}$ None of the materials produced a severe inflammatory response [Table 1].

Regarding the evaluation of differentiation of odontoblasts, one study ${ }^{[14]}$ used cell counting kit-8, alkaline phosphatase (ALP) activity, and quantitative reverse-transcriptase-polymerase chain reaction (qRT-PCR); a second study evaluated osteoblast differentiation by qRT-PCR; ${ }^{[19]}$ and a third study evaluated the odontogenic differentiation, through flow cytometry and scanning electron microscopy (SEM). ${ }^{[9]}$

Regarding cell viability, growth, morphology, and cell adhesion modifications, both materials obtained similar favorable responses. . $2,11,12,16,17,25]$ 
Oliveira, et al.: Biocompatibility of sealers

\begin{tabular}{|c|c|c|c|c|c|c|c|c|c|}
\hline First author & Year & $\begin{array}{l}\text { Type } \\
\text { of } \\
\text { study }\end{array}$ & Type of Sealer & $\begin{array}{l}\text { Type of } \\
\text { laboratorial } \\
\text { analysis }\end{array}$ & $\begin{array}{l}\text { Type of } \\
\text { biocompatibility } \\
\text { test }\end{array}$ & Type of cell & $\begin{array}{l}\text { Animal } \\
\text { or } \\
\text { human }\end{array}$ & $\begin{array}{l}\text { Experimental } \\
\text { period }\end{array}$ & Summary \\
\hline 1. Zhou ${ }^{[9]}$ & 2013 & $\begin{array}{l}\text { In } \\
\text { vitro }\end{array}$ & $\begin{array}{l}\text { MTA (Dentsply } \\
\text { Tulsa Dental, } \\
\text { Tulsa, OK, USA) } \\
\text { Glass ionomer } \\
\text { (GC Fuji IX } \\
\text { GP, Japan) } \\
\text { Biodentine* } \\
\text { (Septodont, } \\
\text { Saint-Maur-des } \\
\text {-Fossés, France) }\end{array}$ & $\begin{array}{l}\text { Flow cytometry, } \\
\text { SEM }\end{array}$ & $\begin{array}{l}\text { Cytotoxicity } \\
\text { Odontogenic } \\
\text { differentiation }\end{array}$ & $\begin{array}{l}\text { Gingival } \\
\text { fibroblast }\end{array}$ & Human & $\begin{array}{l}1,3 \text { and } \\
7 \text { days }\end{array}$ & $\begin{array}{l}\text { Human gingival } \\
\text { fibroblastos } \\
\text { showed similar } \\
\text { response to } \\
\text { extracts from } \\
\text { Biodentine } \\
\text { and MTA as } \\
\text { measured by } \\
\text { cytotoxicity } \\
\text { assay and cell } \\
\text { growth on set } \\
\text { materials }\end{array}$ \\
\hline $\begin{array}{l}\text { 2. Coaguila } \\
\text {-Llerena }{ }^{[10]}\end{array}$ & 2016 & $\begin{array}{l}\text { In } \\
\text { vitro }\end{array}$ & $\begin{array}{l}\text { MTA (Angelus, } \\
\text { Londrina, } \\
\text { PR, Brazil) } \\
\text { ERRM* }^{*} \\
\text { (Brasseler, } \\
\text { Savannah, } \\
\text { GA, USA) } \\
\text { Super EBA } \\
\text { (Bosworth, } \\
\text { Skokie, IL, USA) }\end{array}$ & MTT assay & Cytotoxicity & PDL & Human & $\begin{array}{l}1,3 \text { and } \\
7 \text { days }\end{array}$ & $\begin{array}{l}\text { MTA and ERRM } \\
\text { were less } \\
\text { cytotoxic. The } \\
\text { behavior of all } \\
\text { root end fillings } \\
\text { was similar }\end{array}$ \\
\hline $\begin{array}{l}3 . \\
\text { Willershausen }{ }^{[11]}\end{array}$ & 2013 & $\begin{array}{l}\text { In } \\
\text { vitro }\end{array}$ & $\begin{array}{l}\text { White } \\
\text { MTA (Angelus, } \\
\text { Londrina, } \\
\text { PR, Brazil) } \\
\text { Grey } \\
\text { MTA (Angelus, } \\
\text { Londrina, } \\
\text { PR, Brazil) } \\
\text { ProRoot } \\
\text { MTA (Dentsply/ } \\
\text { Maillefer, } \\
\text { Ballaigues, } \\
\text { Switzerland) } \\
\text { ERRM* } \\
\text { (Brasseler, } \\
\text { Savannah, } \\
\text { GA, USA) }\end{array}$ & $\begin{array}{l}\text { Alamar blue, } \\
\text { fluorescence } \\
\text { staining }\end{array}$ & $\begin{array}{l}\text { Proliferation } \\
\text { cellular } \\
\text { Cellular growth } \\
\text { and morphology }\end{array}$ & $\begin{array}{l}\text { Fibroblasts } \\
\text { and } \\
\text { osteoblasts }\end{array}$ & Human & $\begin{array}{l}6,24,72 \\
\text { and } 96 \mathrm{~h}\end{array}$ & $\begin{array}{l}\text { The root end } \\
\text { materials } \\
\text { ERRM, ProRoot } \\
\text { MTA, and } \\
\text { MTA-angelus } \\
\text { did not } \\
\text { considerably } \\
\text { inhibit the } \\
\text { proliferation of } \\
\text { PDL fibroblasts } \\
\text { and osteoblasts } \\
\text { up to } 96 \text { h, } \\
\text { with ERRM } \\
\text { being the least } \\
\text { inhibitory }\end{array}$ \\
\hline 4. Jiang ${ }^{[12]}$ & 2014 & $\begin{array}{l}\text { In } \\
\text { vitro }\end{array}$ & $\begin{array}{l}\text { iRoot BP Plus* } \\
\text { (Innovative } \\
\text { Bioceramix, } \\
\text { Vancouver, } \\
\text { BC, Canada) } \\
\text { iRoot FS* } \\
\text { (Innovative } \\
\text { Bioceramix. } \\
\text { Vancouver, } \\
\text { BC, Canada) } \\
\text { ProRoot } \\
\text { MTA (Tulsa } \\
\text { Dental, Johnson } \\
\text { City, TN, USA) } \\
\text { Super-EBA } \\
\text { (Bosworth, } \\
\text { Skokie, IL, USA) }\end{array}$ & $\begin{array}{l}\text { MTT assay, } \\
\text { SEM and SEM } \\
\text { analysis }\end{array}$ & $\begin{array}{l}\text { Cytotoxicity } \\
\text { Cellular surface } \\
\text { morphology } \\
\text { Cell adhesion }\end{array}$ & $\begin{array}{l}\text { Osteoblasts } \\
\text { (MG63) }\end{array}$ & Human & $\begin{array}{c}1,3,7, \text { and } \\
14 \text { days }\end{array}$ & $\begin{array}{l}\text { iRoot FS } \\
\text { exhibited } \\
\text { the best cell } \\
\text { adhesion } \\
\text { capacity, and } \\
\text { only Super-EBA } \\
\text { possessed } \\
\text { in vitro } \\
\text { cytotoxicity. } \\
\text { Given the rapid } \\
\text { solidification } \\
\text { of iRoot FS, } \\
\text { this material } \\
\text { showed high } \\
\text { potential for } \\
\text { further clinical } \\
\text { applications }\end{array}$ \\
\hline
\end{tabular}

Contd... 
Oliveira, et al.: Biocompatibility of sealers

\begin{tabular}{|c|c|c|c|c|c|c|c|c|c|}
\hline First author & Year & $\begin{array}{l}\text { Type } \\
\text { of } \\
\text { study }\end{array}$ & Type of Sealer & $\begin{array}{l}\text { Type of } \\
\text { laboratorial } \\
\text { analysis }\end{array}$ & $\begin{array}{l}\text { Type of } \\
\text { biocompatibility } \\
\text { test }\end{array}$ & Type of cell & $\begin{array}{l}\text { Animal } \\
\text { or } \\
\text { human }\end{array}$ & $\begin{array}{l}\text { Experimental } \\
\text { period }\end{array}$ & Summary \\
\hline 5. Ciasca ${ }^{[13]}$ & 2012 & $\begin{array}{l}\text { In } \\
\text { vitro }\end{array}$ & $\begin{array}{l}\text { ProRoot } \\
\text { MTA (MTA; } \\
\text { Dentsply Tulsa } \\
\text { Dental, Johnson } \\
\text { City, TN, USA) } \\
\text { ERRM* (Brasseler, } \\
\text { Savannah, } \\
\text { GA, USA) } \\
\text { AH-26 (Dentsply, } \\
\text { De Trey, } \\
\text { Konstanz, } \\
\text { Germany) }\end{array}$ & $\begin{array}{l}\text { Photomicrograph } \\
\text { images and } \\
\text { qRT-PCR }\end{array}$ & $\begin{array}{l}\text { Cytotoxicity } \\
\text { Cytokine } \\
\text { expression }\end{array}$ & $\begin{array}{l}\text { Osteoblasts } \\
\text { (MG63) }\end{array}$ & Human & $\begin{array}{c}24,36 \text { and } \\
48 \mathrm{~h}\end{array}$ & $\begin{array}{l}\text { ERRM and MTA } \\
\text { showed similar } \\
\text { cytotoxicity } \\
\text { and cytokine } \\
\text { expressions }\end{array}$ \\
\hline 6. Chen ${ }^{[2]}$ & 2016 & $\begin{array}{l}\text { In } \\
\text { vitro }\end{array}$ & $\begin{array}{l}\text { ERRM }^{*} \text { (Brasseler, } \\
\text { Savannah, } \\
\text { GA, USA) } \\
\text { ProRoot } \\
\text { MTA (Dentsply } \\
\text { Tulsa Dental, } \\
\text { Tulsa, OK, USA) }\end{array}$ & $\begin{array}{l}\text { MTT assay } \\
\text { and SEM }\end{array}$ & $\begin{array}{l}\text { Cell proliferation } \\
\text { Cell survival } \\
\text { Cellular surface } \\
\text { morphology }\end{array}$ & $\begin{array}{l}\text { Mesenchymal, } \\
\text { PDL, and } \\
\text { dental pulp } \\
\text { stem cells }\end{array}$ & Human & $\begin{array}{c}1,3,5, \text { and } \\
7 \text { days }\end{array}$ & $\begin{array}{l}\text { MTA and } \\
\text { ERRM are } \\
\text { biocompatible } \\
\text { and promote } \\
\text { cell proliferation } \\
\text { and survival } \\
\text { in an } \\
\text { ERK-signaling } \\
\text { pathway }\end{array}$ \\
\hline 7. Zhang ${ }^{[14]}$ & 2013 & $\begin{array}{l}\text { In } \\
\text { vitro }\end{array}$ & 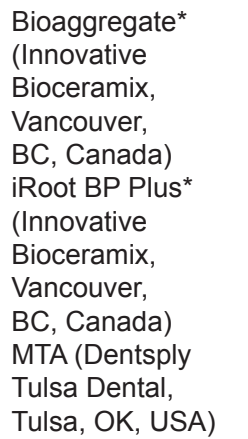 & $\begin{array}{l}\text { CCK-8, ALP } \\
\text { activity, and } \\
\text { qRT-PCR }\end{array}$ & $\begin{array}{l}\text { Cell proliferation } \\
\text { Cell } \\
\text { differentiation } \\
\text { Expression of } \\
\text { odontoblast } \\
\text { differentiation }\end{array}$ & Dental pulp & Human & $\begin{array}{c}1,3,5 \text {, and } \\
7 \text { days }\end{array}$ & $\begin{array}{l}\text { Bioaggregate } \\
\text { and iRoot BP } \\
\text { Plus were } \\
\text { no toxic and } \\
\text { able to induce } \\
\text { mineralization } \\
\text { and } \\
\text { odontoblastic } \\
\text { differentiation }\end{array}$ \\
\hline 8. Jovanovic ${ }^{[15]}$ & 2014 & $\begin{array}{l}\text { In } \\
\text { vitro }\end{array}$ & $\begin{array}{l}\text { Amalgam } \\
\text { (Ekstrakap-D III, } \\
\text { ICN Galenika, } \\
\text { Serbia) } \\
\text { MTA (Angelus, } \\
\text { Londrina, } \\
\text { PR, Brazil) } \\
\text { Biodentine* } \\
\text { (Septodont, Saint } \\
\text { Maur-des-Fossés, } \\
\text { France) }\end{array}$ & $\begin{array}{l}\text { DET, MTT } \\
\text { assay, and agar } \\
\text { diffusion test }\end{array}$ & Cytotoxicity & $\begin{array}{l}\text { Fibroblasts } \\
\text { (MRC-5 and } \\
\text { mouse L929) }\end{array}$ & $\begin{array}{l}\text { Human } \\
\text { and } \\
\text { animal }\end{array}$ & $\begin{array}{l}24,48, \\
\text { and } 72 \mathrm{~h}\end{array}$ & $\begin{array}{l}\text { Biocompatibility } \\
\text { tests showed } \\
\text { high level of cell } \\
\text { compatibility } \\
\text { of all the three } \\
\text { tested materials }\end{array}$ \\
\hline 9. $\mathrm{Ma}^{[16]}$ & 2011 & $\begin{array}{l}\text { In } \\
\text { vitro }\end{array}$ & $\begin{array}{l}\text { ERRM* (Brasseler, } \\
\text { Savannah, } \\
\text { GA, USA) } \\
\text { MTA (Dentsply } \\
\text { Tulsa Dental, } \\
\text { Tulsa, OK, USA) } \\
\text { IRM (Dentsply } \\
\text { Caulk, Milford, } \\
\text { DE) } \\
\text { Cavit G (3M ESPE } \\
\text { AG, Seefeld, } \\
\text { Germany) }\end{array}$ & $\begin{array}{l}\text { MTT assay } \\
\text { and scanning } \\
\text { electron } \\
\text { microscope }\end{array}$ & $\begin{array}{l}\text { Cytotoxicity } \\
\text { Cell viability } \\
\text { Cell adhesion }\end{array}$ & Fibroblasts & Human & $\begin{array}{l}1,3 \text { and } \\
7 \text { days }\end{array}$ & $\begin{array}{l}\text { ERRM putty } \\
\text { and paste } \\
\text { displayed } \\
\text { similar in vitro } \\
\text { biocompatibility } \\
\text { to MTA }\end{array}$ \\
\hline
\end{tabular}

Contd... 
Oliveira, et al.: Biocompatibility of sealers

\begin{tabular}{|c|c|c|c|c|c|c|c|c|c|}
\hline First author & Year & $\begin{array}{l}\text { Type } \\
\text { of } \\
\text { study }\end{array}$ & Type of Sealer & $\begin{array}{l}\text { Type of } \\
\text { laboratorial } \\
\text { analysis }\end{array}$ & $\begin{array}{l}\text { Type of } \\
\text { biocompatibility } \\
\text { test }\end{array}$ & Type of cell & $\begin{array}{l}\text { Animal } \\
\text { or } \\
\text { human }\end{array}$ & $\begin{array}{l}\text { Experimental } \\
\text { period }\end{array}$ & Summary \\
\hline $\begin{array}{l}\text { 10. Corral } \\
\text { Nuñez } z^{[17]}\end{array}$ & 2014 & $\begin{array}{l}\text { In } \\
\text { vitro }\end{array}$ & $\begin{array}{l}\text { Biodentine* } \\
\text { (Septodont, } \\
\text { Saint Maur de } \\
\text { Fosses, France) } \\
\text { ProRoot MTA } \\
\text { (Dentsply } \\
\text { Endodontics, } \\
\text { Tulsa, OK) }\end{array}$ & $\begin{array}{l}\text { Alamar blue, } \\
\text { SEM, and } \\
\text { qRT-PCR }\end{array}$ & $\begin{array}{l}\text { Cell viability } \\
\text { Morphology } \\
\text { cellular } \\
\text { Cytokine } \\
\text { expression }\end{array}$ & $\begin{array}{l}\text { Fibroblasts } \\
\text { 3T3 }\end{array}$ & Animal & $\begin{array}{l}3,6,24 \\
\text { and } 72 \mathrm{~h}\end{array}$ & $\begin{array}{l}\text { Biodentine } \\
\text { and MTA } \\
\text { showed similar } \\
\text { cytotoxicity } \\
\text { and induced a } \\
\text { similar pattern } \\
\text { of cytokine } \\
\text { expression }\end{array}$ \\
\hline 11. Khalili ${ }^{[18]}$ & 2015 & In vivo & $\begin{array}{l}\text { MTA (Dentsply } \\
\text { Tulsa Dental, } \\
\text { Tulsa, OK) } \\
\text { ERRM* }^{*} \\
\text { (Brasseler, } \\
\text { Savannah, } \\
\text { GA, USA) }\end{array}$ & $\begin{array}{l}\text { Histological } \\
\text { evaluation }\end{array}$ & Tissue reactions & Subcutaneous & $\begin{array}{l}\text { Animal } \\
\text { (Wistar } \\
\text { rats) }\end{array}$ & $\begin{array}{l}7 \text { and } \\
30 \text { days }\end{array}$ & $\begin{array}{l}\text { Both ERRM } \\
\text { and MTA cause } \\
\text { an injurious } \\
\text { effect when } \\
\text { implanted in rat } \\
\text { subcutaneous } \\
\text { tissues after } 7 \\
\text { and } 30 \text { days, } \\
\text { ERRM is } \\
\text { significantly } \\
\text { less injurious } \\
\text { to tissues } \\
\text { than MTA }\end{array}$ \\
\hline 12. Rifaey ${ }^{[19]}$ & 2016 & $\begin{array}{l}\text { In } \\
\text { vitro }\end{array}$ & $\begin{array}{l}\text { ProRoot } \\
\text { MTA (Dentsply } \\
\text { Tulsa Dental } \\
\text { Specialties, } \\
\text { Tulsa, OK) } \\
\text { ERRM* } \\
\text { (Brasseler, } \\
\text { Savannah, } \\
\text { GA, USA) }\end{array}$ & qRT-PCR & $\begin{array}{l}\text { Osteoblast } \\
\text { differentiation }\end{array}$ & Osteoblasts & Animal & $\begin{array}{l}7,14, \text { and } \\
21 \text { days }\end{array}$ & $\begin{array}{l}\text { ERRM } \\
\text { promotes } \\
\text { osteoblast } \\
\text { differentiation } \\
\text { better than } \\
\text { MTA and } \\
\text { controls with no } \\
\text { material in three } \\
\text { dimensions }\end{array}$ \\
\hline 13. De-Deus ${ }^{[20]}$ & 2012 & $\begin{array}{l}\text { In } \\
\text { vitro }\end{array}$ & $\begin{array}{l}\text { iRoot BP Plus* } \\
\text { (Innovative } \\
\text { Bioceramix, } \\
\text { Vancouver, } \\
\text { BC, Canada) } \\
\text { ProRoot MTA } \\
\text { (Dental, Tulsa, } \\
\text { Ok, USA) }\end{array}$ & $\begin{array}{l}\mathrm{XTT}, \mathrm{NR} \\
\text { and CVDE }\end{array}$ & Cytotoxicity & Osteoblast & Human & 24 or $48 \mathrm{~h}$ & $\begin{array}{l}\text { iRoot BP Plus } \\
\text { and MTA were } \\
\text { biocompatible } \\
\text { and did not } \\
\text { induce critical } \\
\text { cytotoxic effects }\end{array}$ \\
\hline $\begin{array}{l}14 . \\
\text { Modareszadeh }\end{array}$ & 2012 & $\begin{array}{l}\text { In } \\
\text { vitro }\end{array}$ & $\begin{array}{l}\text { ERRM* } \\
\text { (Brasseler, } \\
\text { Savannah, } \\
\text { GA, USA) } \\
\text { ProRoot } \\
\text { MTA (Dentsply } \\
\text { Tulsa Dental, } \\
\text { Tulsa, OK) }\end{array}$ & $\begin{array}{l}\text { MTS and } \\
\text { p-NPP assay }\end{array}$ & Cytotoxicity & $\begin{array}{l}\text { Saos-2 } \\
\text { Osteoblast } \\
\text {-like }\end{array}$ & Human & $\begin{array}{l}1,3 \text { and } \\
7 \text { days }\end{array}$ & $\begin{array}{l}\text { Elutes of ERRM } \\
\text { significantly } \\
\text { reduced the } \\
\text { bioactivity and } \\
\text { ALP activity of } \\
\text { Saos- } 2 \text { human } \\
\text { osteoblast-like } \\
\text { cells. MTA did } \\
\text { not affect the } \\
\text { cells' bioactivity } \\
\text { or ALP activity }\end{array}$ \\
\hline 15. Damas ${ }^{[22]}$ & 2011 & $\begin{array}{l}\text { In } \\
\text { vitro }\end{array}$ & $\begin{array}{l}\text { MTA (Angelus, } \\
\text { Londrina, } \\
\text { PR, Brazil) } \\
\text { ERRM* } \\
\text { (Brasseler, } \\
\text { Savannah, } \\
\text { GA, USA) }\end{array}$ & MTT assay & Cytotoxicity & Fibroblasts & Human & $24 \mathrm{~h}$ & $\begin{array}{l}\text { The ERRM } \\
\text { was shown to } \\
\text { have similar } \\
\text { cytotoxicity } \\
\text { levels to those } \\
\text { of ProRoot } \\
\text { MTA and MTA }\end{array}$ \\
\hline
\end{tabular}

Contd... 
Oliveira, et al.: Biocompatibility of sealers

\begin{tabular}{|c|c|c|c|c|c|c|c|c|c|}
\hline First author & Year & $\begin{array}{l}\text { Type } \\
\text { of } \\
\text { study }\end{array}$ & Type of Sealer & $\begin{array}{l}\text { Type of } \\
\text { laboratorial } \\
\text { analysis }\end{array}$ & $\begin{array}{l}\text { Type of } \\
\text { biocompatibility } \\
\text { test }\end{array}$ & Type of cell & $\begin{array}{l}\text { Animal } \\
\text { or } \\
\text { human }\end{array}$ & $\begin{array}{l}\text { Experimental } \\
\text { period }\end{array}$ & Summary \\
\hline $\begin{array}{l}16 . \\
\text { Samyuktha }{ }^{[23]}\end{array}$ & 2014 & $\begin{array}{l}\text { In } \\
\text { vitro }\end{array}$ & $\begin{array}{l}\text { MTA (Dentsply } \\
\text { Tulsa Dental, } \\
\text { Tulsa, OK) } \\
\text { ERRM** }^{*} \\
\text { (Brasseler, }_{\text {Savannah, }} \\
\text { GA, USA) } \\
\text { Biodentine* } \\
\text { (Septodont, } \\
\text { Saint Maur de } \\
\text { Fosses, France) }\end{array}$ & $\begin{array}{l}\text { Trypan blue } \\
\text { dye assay }\end{array}$ & Cytotoxicity & Fibroblasts & Human & 24 and $48 \mathrm{~h}$ & $\begin{array}{l}\text { MTA was } \\
\text { shown to be } \\
\text { less toxic to } \\
\text { PDL fibroblasts } \\
\text { than ERRM } \\
\text { and Biodentine }\end{array}$ \\
\hline 17. Hirschman ${ }^{[24]}$ & 2012 & $\begin{array}{l}\text { In } \\
\text { vitro }\end{array}$ & $\begin{array}{l}\text { White } \\
\text { MTA (Angelus, } \\
\text { Londrina, } \\
\text { PR, Brazil) } \\
\text { ERRM* (Brasseler, }_{\text {Savannah, USA) }} \\
\text { Dycal (Dentsply } \\
\text { De Tray GmbH, } \\
\text { Konstanz, } \\
\text { Germany) } \\
\text { UBP (Ultradent } \\
\text { Products, Inc., } \\
\text { South Jordan, UT) }\end{array}$ & MTT assay & Cytotoxicity & Fibroblasts & Human & $\begin{array}{c}2,5 \text {, and } \\
8 \text { days }\end{array}$ & $\begin{array}{l}\text { MTA, ERRM, } \\
\text { and UBP had } \\
\text { statistically } \\
\text { similar adult } \\
\text { human dermal } \\
\text { fibroblast } \\
\text { cytotoxicity } \\
\text { levels. Relative } \\
\text { to the negative } \\
\text { control, only } \\
\text { Dycal was } \\
\text { show to have } \\
\text { a statistically } \\
\text { significant } \\
\text { cytotoxic } \\
\text { effect to adult } \\
\text { human dermal } \\
\text { fibroblasts at } \\
\text { all tested }\end{array}$ \\
\hline 18. $L v^{[25]}$ & 2017 & $\begin{array}{l}\text { In } \\
\text { vitro }\end{array}$ & $\begin{array}{l}\text { iRoot } \\
\text { FS* (Innovative } \\
\text { Bioceramix, } \\
\text { Vancouver, BC, } \\
\text { Canada) } \\
\text { iRoot BP } \\
\text { Plus* (Innovative } \\
\text { Bioceramix, } \\
\text { Vancouver, BC, } \\
\text { Canada) } \\
\text { ProRoot } \\
\text { MTA (Dentsply } \\
\text { Tulsa Dental, } \\
\text { Tulsa, OK, USA) }\end{array}$ & $\begin{array}{l}\text { Kit-8, annexin } \\
\text { V-FITC and } \\
\text { propidium } \\
\text { iodide flow, } \\
\text { fluorescence } \\
\text { microscope } \\
\text { and scanning } \\
\text { electron } \\
\text { microscope }\end{array}$ & $\begin{array}{l}\text { Cell viability } \\
\text { Cellular } \\
\text { morphology } \\
\text { Cell attachment }\end{array}$ & МС3Т3-E1 & Animal & $\begin{array}{c}24 \mathrm{~h}, 1,2 \text { and } \\
3 \text { days }\end{array}$ & $\begin{array}{l}\text { iRoot FS and } \\
\text { iRoot BP Plus } \\
\text { in the set form } \\
\text { promoted the } \\
\text { viability of } \\
\text { MC3T3-E1 } \\
\text { osteoblast cells. } \\
\text { This finding is } \\
\text { similar to that } \\
\text { observed in } \\
\text { MTA }\end{array}$ \\
\hline \multicolumn{10}{|c|}{$\begin{array}{l}\text { "calcium silicate-based materials MTA: Mineral trioxide aggregate, ERRM: Endosequence root repair material, PDL: Periodontal ligament, SEM: Scanning } \\
\text { electron microscopy, qRT-PCR: Quantitative reverse-transcriptase-polymerase chain reaction, CCK: Cell counting kit, ALP: Alkaline phosphatase, NR: } \\
\text { Neutral red, CVDE: Crystal violet dye elution, p-NPP: P-nitrophenyl phosphate, UBP: Ultra-blend Plus, MTT: Colorimetric Test with Tetrazolium Salt } \\
\text { (3-(4,5-dimethylthiazol-2-yl)-2,5-diphenyl-2H-tetrazolium bromide), DET:Dye exclusion test, MTS: methylthiazol sulfophenyl (3-(4,5-dimethylthiazol-2- } \\
\text { yl)-5-(3-carboxymethoxyphenyl)-2-(4-sulfophenyl)-2H-tetrazolium, XTT: 2,3-bis-(2-methoxy-4-nitro-5-sulphenyl)-(2H)-tetrazolium-5-carboxanilide) }\end{array}$} \\
\hline
\end{tabular}

\section{DISCUSSION}

Few studies have compared the cytocompatibility and cell interactions between bioceramic materials and MTA and other conventional sealers, such as containing calcium hydroxide, zinc oxide and eugenol, and resins. Based on the current literature, there are no systematic reviews that make this kind of comparison.

The cytotoxic potential is one of the most common features investigated in in vitro studies to determine the biocompatibility of root repair materials before testing them in clinical studies. The cytotoxicity of materials can be due to the presence of toxic or soluble compounds in their composition. Jiang et al. ${ }^{[12]}$ compared the cytotoxicity in vitro of iRoot BP Plus, iRoot FS, ProRoot MTA, and Super-EBA in fibroblasts and human osteoblasts. All materials, with the exception of Super-EBA, exhibited insignificant cytotoxicity. In addition, iRoot FS demonstrated great potential in other clinical 
applications due to its rapid setting time $(1 \mathrm{~h})$ and better cell adhesion capacity when compared to other studied materials.

Rifaey et al., ${ }^{[19]}$ investigating the gene expression levels of bone sialoprotein, ALP, and Osterix, found that endosequence root repair material (ERRM) increased the differentiation of osteoblasts when compared to MTA. The results obtained for the bioceramics can be explained by the presence of nontoxic compounds in their composition, including calcium and phosphorus. ${ }^{[12]}$ The biocompatibility of these materials can be attributed to the formation of hydroxyapatite in the presence of $\mathrm{Ca}^{2+}$ ion during the setting reaction. ${ }^{[26]}$ Another explanation for this result, according to the study, was the use of a not so frequent biocompatibility test, the three-dimensional culture system (Alvetex scaffold), which best resembles clinical conditions, where the cells are not placed in contact with materials. Instead, they are attracted to the proximity of the studied material. ${ }^{[19]}$

On the other hand, in the study of Modareszadeh et al., ${ }^{[21]}$ ERRM significantly reduced the bioactivity of human osteoblasts. Samyuktha et al. ${ }^{[23]}$ found that MTA was less toxic to periodontal ligament fibroblasts than ERRM and biodentine. The bioactivity of the MTA is dependent on its high $\mathrm{pH}$, which results in the release of calcium ions after setting. ${ }^{[27]}$ These divergent results may be due to differences in the cell lines (osteoblasts and fibroblasts), type of laboratory analysis (MTT assay, Trypan blue dye assay), concentrations and dilutions, and duration of the experiments used to evaluate these bioceramic materials (ERRM and biodentine).

The contact of cells with the surface of the material is a good indicator that the materials are biocompatible. In addition, if the materials stimulate cell proliferation or survival, they are likely to promote the repair process. ${ }^{[28]}$ In many studies, bioceramic materials promoted cell proliferation and viability and their performance was similar or better than that of MTA. In contrast, De-Deus et al. ${ }^{[20]}$ found clear differences in cell viability in the XTT assay: tetrazolium dye 2,3-bis(2-methoxy-4-nitro-5-sulphenyl)-(2H)-tetrazolium5-carboxanilide between iRoot BP Plus and ProRoot MTA after $48 \mathrm{~h}$ of exposure. The bioceramic material reduced cell viability significantly more than that observed in the other groups (zinc oxide- and eugenol-containing cement and control, in which the cells were exposed to unconditioned medium). However, iRoot BP Plus did not induce critical cytotoxic effects. Thus, the biocompatibility of the bioceramic sealer was comparable to that of MTA.

With respect to morphology and the capacity of root canal sealers to promote cell adhesion, bioceramic materials were also found to be similar or better than MTA. In the study of Jiang et al., ${ }^{[12]}$ iRoot FS promoted better cell-cell adhesion (L929 and MG63). Previous studies have shown that cell adhesion is highly dependent on cell morphology and on the surface characteristics of the materials. ${ }^{[28,29]}$ These characteristics influence the behavior, migration, adhesion, and differentiation of cells. ${ }^{[28]}$

Chen et al. ${ }^{[2]}$ found only minimal differences between the surface characteristics of MTA and ERRM by SEM. Ma et al. ${ }^{[16]}$ still visually confirmed, through the same method, a positive cellular interaction between the two cement. Both materials promoted cell proliferation and survival. The similar granular surface characteristics of MTA and ERRM may therefore explain the similar biological activities of these materials.

On the other hand, Corral Nuñez et al. ${ }^{[17]}$ found changes in cell viability during fibroblast exposure to biodentine and MTA. After $24 \mathrm{~h}$ of exposure to biodentine, the study showed an increase in cell viability, which was not observed with MTA. This result can be explained by differences in the composition between the two materials, such as the presence of the radiopacifier. The radiopacifier for MTA is bismuth oxide. Its use has been questioned for not promoting cell growth. In addition, calcium phosphate crystals, produced by the reaction of MTA with the SEM preparation, can generate distorted images. However, over time, it appears that the cells can repair themselves. Although SEM is the most widely used method for assessing cell viability in direct contact with calcium silicate-based materials, studies have shown that sample processing may affect morphology and consequent cell viability ${ }^{[30]}$ and may contribute to the controversial results.

Regarding inflammatory responses and the production of pro-inflammatory mediators and cytokines, Ciasca et al. ${ }^{[13]}$ reported similar effects for ERRM putty, flow, and ProRoot MTA, including the expression of IL-1b, IL-6, and IL-8 in all samples with minimal expression of TNF- $\alpha$ determined by RT-PCR. A slight difference was observed between the levels of cytokine expression, in which the ERRM putty showed higher levels during the first $24 \mathrm{~h}$. One explanation for this would be based on the use of different types of vehicles applied 
to create ERRM flow, although the difference was not significant. Many studies have shown that the interaction between the components of bioceramics and osteoblasts increases the production of cytokines, such as ILs and TNF. The high expression of these bone resorption cytokines has a beneficial effect on bone formation. Thus, these materials present high repairing potential.

In contrast to most studies that reported similar characteristics for bioceramic materials and MTA, Khalil and Abunasef ${ }^{[18]}$ showed more tissue injury with MTA than ERRM, after their applications in rat subcutaneous tissues. The authors observed areas of necrosis and abscesses that were attributed to the setting of MTA. An exothermic reaction occurs during this process in which tissues are exposed to high temperatures that can cause ischemia, cell death, and tissue necrosis.

One of the challenges of this research was the lack of standardization of the biocompatibility tests and evaluation times used in each study between the bioceramic materials and MTA. Different methods were likely responsible for the conflicting results. It is very important that root canal sealers exhibit acceptable biocompatibility and cytotoxicity and good biological properties. Therefore, the hypothesis tested in this review was not accepted, since the bioceramic materials showed biological properties similar to those of MTA such as good biocompatibility indicated by low cytotoxicity as well as the induction of cell proliferation and adhesion, adequate expression of inflammatory cytokines, and reduced pulp inflammation after the acute phase.

\section{CONCLUSION}

This systematic review therefore suggests that the choice of repair bioceramic materials or MTA based on biocompatibility should be the professional's decision.

\section{Financial support and sponsorship}

Nil.

\section{Conflicts of interest}

There are no conflicts of interest.

\section{REFERENCES}

1. Bernáth $\mathrm{M}$, Szabó J. Tissue reaction initiated by different sealers. Int Endod J 2003;36:256-61.

2. Chen I, Salhab I, Setzer FC, Kim S, Nah HD. A new calcium silicate-based bioceramic material promotes human osteo- and odontogenic stem cell proliferation and survival via the extracellular signal-regulated kinase signaling pathway. J Endod 2016;42:480-6.

3. Xu P, Liang J, Dong G, Zheng L, Ye L. Cytotoxicity of RealSeal on human osteoblast-like MG63 cells. J Endod 2010;36:40-4.

4. Desai S, Chandler N. Calcium hydroxide-based root canal sealers: A review. J Endod 2009;35:475-80.

5. Alanezi AZ, Jiang J, Safavi KE, Spangberg LS, Zhu Q. Cytotoxicity evaluation of endosequence root repair material. Oral Surg Oral Med Oral Pathol Oral Radiol Endod 2010;109:e122-5.

6. Zhang W, Li Z, Peng B. Assessment of a new root canal sealer's apical sealing ability. Oral Surg Oral Med Oral Pathol Oral Radiol Endod 2009;107:e79-82.

7. Chen CL, Kao CT, Ding SJ, Shie MY, Huang TH. Expression of the inflammatory marker cyclooxygenase- 2 in dental pulp cells cultured with mineral trioxide aggregate or calcium silicate cements. J Endod 2010;36:465-8.

8. Hung CJ, Kao CT, Shie MY, Huang TH. Comparison of host inflammatory responses between calcium-silicate base material and intermediate restorative material. J Dent Sci 2014;9:158-64.

9. Zhou HM, Shen Y, Wang ZJ, Li L, Zheng YF, Häkkinen L, et al. In vitro cytotoxicity evaluation of a novel root repair material. J Endod 2013;39:478-83.

10. Coaguila-Llerena H, Vaisberg A, Velásquez-Huamán Z. In vitro cytotoxicity evaluation of three root-end filling materials in human periodontal ligament fibroblasts. Braz Dent J 2016;27:187-91.

11. Willershausen I, Wolf T, Kasaj A, Weyer V, Willershausen B, Marroquin BB, et al. Influence of a bioceramic root end material and mineral trioxide aggregates on fibroblasts and osteoblasts. Arch Oral Biol 2013;58:1232-7.

12. Jiang $Y$, Zheng $Q$, Zhou $X$, Gao $Y$, Huang D. A comparative study on root canal repair materials: A cytocompatibility assessment in L929 and MG63 cells. ScientificWorldJournal 2014;2014:463826.

13. Ciasca M, Aminoshariae A, Jin G, Montagnese T, Mickel A. A comparison of the cytotoxicity and proinflammatory cytokine production of EndoSequence root repair material and ProRoot mineral trioxide aggregate in human osteoblast cell culture using reverse-transcriptase polymerase chain reaction. J Endod 2012;38:486-9.

14. Zhang S, Yang X, Fan M. BioAggregate and iRoot BP plus optimize the proliferation and mineralization ability of human dental pulp cells. Int Endod J 2013;46:923-9.

15. Jovanovic L, Miljevic I, Petrovic B, Markovic D, Kojic V, Bajkin B. Biocompatibility of three root end filling materials. J Biomater Tissue Eng 2014;4:1-5.

16. Ma J, Shen Y, Stojicic S, Haapasalo M. Biocompatibility of two novel root repair materials. J Endod 2011;37:793-8.

17. Corral Nuñez CM, Bosomworth HJ, Field C, Whitworth JM, Valentine RA. Biodentine and mineral trioxide aggregate induce similar cellular responses in a fibroblast cell line. J Endod 2014;40:406-11.3

18. Khalil WA, Abunasef SK. Can mineral trioxide aggregate and nanoparticulate EndoSequence root repair material produce injurious effects to rat subcutaneous tissues? J Endod 2015;41:1151-6.

19. Rifaey HS, Villa M, Zhu Q, Wang YH, Safavi K, Chen IP, et al. Comparison of the osteogenic potential of Mineral trioxide aggregate and endosequence Root repair material in a 3-dimensional culture system. J Endod 2016;42:760-5.

20. De-Deus G, Canabarro A, Alves GG, Marins JR, Linhares AB, Granjeiro JM, et al. Cytocompatibility of the ready-to-use bioceramic putty repair cement iRoot BP plus with primary human osteoblasts. Int Endod J 2012;45:508-13.

21. Modareszadeh MR, Di Fiore PM, Tipton DA, Salamat N. Cytotoxicity and alkaline phosphatase activity evaluation of endosequence root repair material. J Endod 2012;38:1101-5.

22. Damas BA, Wheater MA, Bringas JS, Hoen MM. Cytotoxicity comparison of mineral trioxide aggregates and EndoSequence bioceramic root repair materials. J Endod 2011;37:372-5.

23. Samyuktha V, Ravikumar P, Nagesh B, Ranganathan K, Jayaprakash $T$, Sayesh V, et al. Cytotoxicity evaluation of root repair materials in human-cultured periodontal ligament fibroblasts. J Conserv Dent 2014;17:467-70.

24. Hirschman WR, Wheater MA, Bringas JS, Hoen MM. Cytotoxicity comparison of three current direct pulp-capping agents with a new bioceramic root repair putty. J Endod 2012;38:385-8.

25. Lv F, Zhu L, Zhang J, Yu J, Cheng X, Peng B, et al. Evaluation of the in vitro biocompatibility of a new fast-setting ready-to-use root filling 
Oliveira, et al.: Biocompatibility of sealers

and repair material. Int Endod J 2017;50:540-8.

26. Güven EP, Taşlı PN, Yalvac ME, Sofiev N, Kayahan MB, Sahin F, et al. In vitro comparison of induction capacity and biomineralization ability of mineral trioxide aggregate and a bioceramic root canal sealer. Int Endod J 2013;46:1173-82.

27. Hansen SW, Marshall JG, Sedgley CM. Comparison of intracanal EndoSequence root repair material and ProRoot MTA to induce $\mathrm{pH}$ changes in simulated root resorption defects over 4 weeks in matched pairs of human teeth. J Endod 2011;37:502-6.
28. Roach P, Eglin D, Rohde K, Perry CC. Modern biomaterials: A review - Bulk properties and implications of surface modifications. J Mater Sci Mater Med 2007;18:1263-77.

29. Berry CC, Campbell G, Spadiccino A, Robertson M, Curtis AS. The influence of microscale topography on fibroblast attachment and motility. Biomaterials 2004;25:5781-8.

30. Camilleri J, Montesin FE, Papaioannou S, McDonald F, Pitt Ford TR. Biocompatibility of two commercial forms of mineral trioxide aggregate. Int Endod J 2004;37:699-704. 